\title{
Leishmaniases in Greece
}

\author{
Pantelis Ntais, Dimitra Sifaki-Pistola, Vasiliki Christodoulou, Ippokratis Messaritakis, Francine Pratlong, \\ George Poupalos, and Maria Antoniou* \\ Laboratory of Clinical Bacteriology, Parasitology, Zoonoses and Geographical Medicine, Faculty of Medicine, University of Crete, Crete, Greece; \\ Veterinary Services of Cyprus, Nicosia, Cyprus; University Montpellier 1, Faculty of Medicine, Laboratory of Parasitology-Mycology, \\ Hospital University Centre (CHRU) of Montpellier and French National Reference Centre for Leishmaniases, \\ CNRS 5290 - IRD 224 - University Montpellier 1\&2 (UMR “MIVEGEC”)
}

\begin{abstract}
During the last 35 years, visceral leishmaniasis has spread in Greece with autochthonous human cases appearing in 41 of the 54 prefectures. The occurrence of the disease was mapped and related to dog seropositivity, environmental and geospatial risk factors. Average dog seropositivity was $22.1 \%$ and positive animals were found in 43 of 54 prefectures. Factors like: altitude, presence of water bodies, land use, wind speed, mean land surface temperature, mean relative humidity, and mean annual rainfall were found to affect dog seropositivity. Cases of cutaneous leishmaniasis, caused by Leishmania tropica are also increasing. Phlebotomus similis believed to be the potential vector of $L$. tropica in Greece, was found in areas where the disease is widespread but also where cases have never been reported implying a danger of introduction of this anthroponotic parasite to new regions.
\end{abstract}

\section{INTRODUCTION}

Leishmaniasis, an important but neglected disease caused by the protozoan parasite Leishmania, is found in 98 countries worldwide. ${ }^{1}$ Natural transmission may be zoonotic or anthroponotic, depending on the parasite species and the geographical location, and it takes place by the bite of blood sucking phlebotomine sandflies. In the Mediterranean basin four Leishmania species are found causing visceral (VL) or/and cutaneous disease (CL): Leishmania infantum, the most common species in this area, responsible for VL and sporadic CL cases in humans and visceral disease in dogs (CanL) is an important zoonotic pathogen using the dog as reservoir host; Leishmania major, found in North Africa and Middle East, causes zoonotic CL; Leishmania tropica, with a limited presence in Europe, causes anthroponotic $\mathrm{CL}^{2,3}$; and Leishmania donovani, recently reported in Cyprus, causes anthroponotic VL and CL. ${ }^{4,5}$

Although leishmaniasis is considered a tropical and subtropical disease, it has become endemic in other geographical areas and $L$. infantum is reported to be spreading to parts of Central Europe, like northern Italy, Hungary, and southern Germany. ${ }^{6-8}$ This spread is related to factors concerning environmental, demographic, and human behavioral changes. ${ }^{9,10}$ The emergence and spread of human immunodeficiency virus (HIV) aids in the spread of leishmaniasis because this co-infection alters the epidemiology of the disease in favor of the parasite. At the same time, the emergence of parasite resistance to meglumine antimoniate and possible sandfly resistance to current insecticides is making the battle against this public health problem even more difficult.

Dog seropositivity is reported to have reached $48 \%$ in many endemic areas in Southern Europe where it constitutes a major veterinary problem. ${ }^{11}$ However, a great proportion of seropositive dogs may be asymptomatic and so they may be overlooked. The development of vaccines against the disease in the dog (the first one available since 2011), will help

* Address correspondence to Maria Antoniou, Laboratory of Clinical Bacteriology, Parasitology, Zoonoses and Geographical Medicine, Faculty of Medicine, University of Crete, Voutes, Heraklion, Crete 71003, Greece. E-mail: antoniou@med.uoc.gr in controlling the disease in the reservoir and, as a consequence, in the human population as well.

Visceral leishmaniasis in the Mediterranean region was first reported in the Greek island of Spetses in $1835 .{ }^{12}$ Until the 1940s, the visceral and the cutaneous form of the disease were spread in many areas of Greece where it was known under several colloquial names. The use of DDT, to combat malaria during World War II, diminished the frequency of mosquito and sandfly transmitted diseases. However, in the last 35 years, the disease has re-emerged with an increasing number of human and dog cases per year. An average of 47 leishmaniasis cases are reported annually (data from the Hellenic Center for Disease Control and Prevention [HCDCP]) a number which, it is emphasized, is an underestimation of the true situation although leishmaniasis is a notifiable disease in the country. Interestingly, human leishmaniasis cases are now reported from all parts of the country (HCDCP), which is either caused by the geographical spread of the disease or the improvement of reporting procedures. Nonetheless, veterinary records substantiate the emergence of CanL in new areas and the increase in dog cases in areas where the problem was already evident. Thirteen sandfly species, 10 belonging to the medically important genus of Phlebotomus and 3 to Sergentomyia are known in Greece, ${ }^{13-17} 5$ of which are proven or suspected vectors of $L$. infantum ( $P$. perfiliewi, P. tobbi, P. neglectus), L. donovani (P. tobbi), L. tropica (P. similis), and L. major (P. papatasi).

The aim of this work was to investigate the situation in Greece regarding leishmaniasis as it stands today: the geographical distribution of VL, CL, CanL, Leishmania species and zymodemes as well as sandfly species and relate the distribution of the disease to epidemiological and environmental factors using geospatial tools.

\section{MATERIALS AND METHODS}

Study area. The study was conducted in all 54 prefectures of Greece, situated in southeastern Europe, of $131,957 \mathrm{~km}^{2}$ and 10,964,020 inhabitants (Hellenic Statistical Authority, 2010). Approximately 700,000 dogs and 200,000 stray dogs are believed to live in the country (data from veterinary companies operating in Greece). Greece has the twelfth longest coastline 
in the world, (13,676 km long) and over 1,400 islands, of which 227 are inhabited; $80 \%$ of the terrain is mountainous constituting an obstacle for the natural geographical spread of sandflies in many regions (Figure 1).

Human cases. A number of patients, registered in different hospitals (all hospitals in Crete and five from Attica, where the majority of the cases occur) with persisting fever of unknown etiology or suspicious skin lesions, were tested in our laboratory at the Faculty of Medicine, University of Crete. A VL case was diagnosed by serology (any antibody titer $\geq 1 / 160$ was considered suspicious and further tests were performed to confirm the infection), polymerase chain reaction (PCR) and culture on available biological material. At the same time, when bone marrow aspirate was available, Giemsa stained preparations were checked for the presence of amastigotes in macrophages, using light microscopy. A human CL case was confirmed by PCR and culture of skin tissue and blood; serology was also performed for the detection of antibodies which, however, was not used for diagnostic purposes but to complete the picture of the case. Clinical and epidemiological information (including address of residence) was collected from each patient and the patient's written

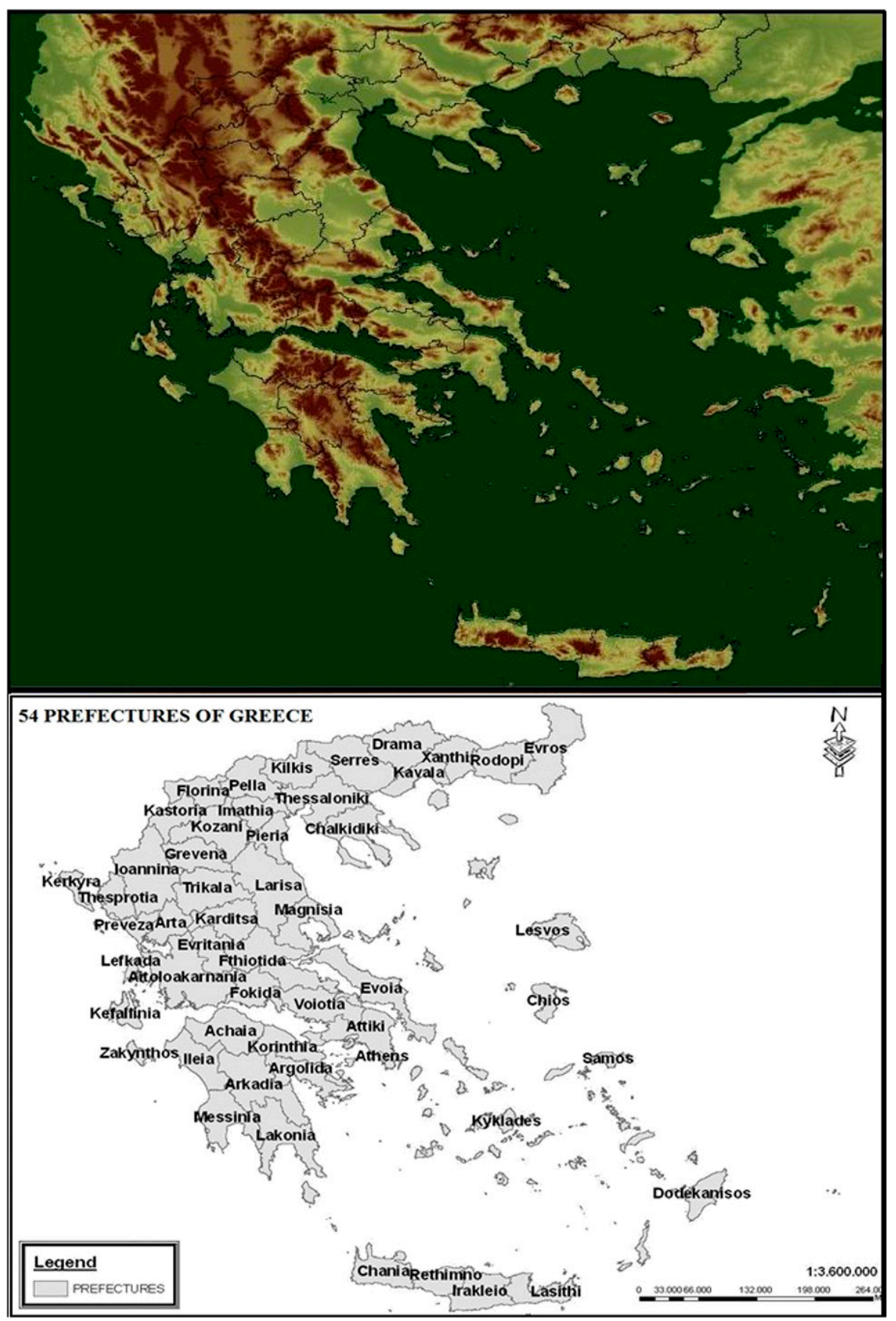

FIGURE 1. Map of Greece showing the terrain of the country and the 54 prefectures. 
consent on the use of the biological material collected. After treatment, antibody response of VL patients, was monitored for at least 1 year until it dropped considerably, to avoid relapses. Data of the HCDCP for the years 2005-2010 were also used in this study; they concerned 222 patients.

Dog epidemiological study. During 2005-2010, a network was established with veterinarians working in the country. They were asked to complete a questionnaire regarding CanL in their area of practice and to provide dog samples from animals visiting their clinic for any reason: vaccination, hair cut, nail cut, deworming, general check up, treatments, and other purposes, without discrimination. Biological samples, from stray dogs, were also provided by veterinarians working in dog kennels. Such animals are taken to kennels for up to 2 weeks to be vaccinated, given antiparasitic pills, or to be castrated before they are again released to the neighborhood where they were found. Veterinarians from 43 prefectures (63 different veterinary clinics) responded to our request and provided samples from 5,772 randomly selected dogs. The animals were examined clinically and biological material was collected, after the written consent of the owner, and questionnaires with personal, epidemiological, and clinical data for each dog were completed. Peripheral blood samples (whole blood and without EDTA from all dogs taking part in the study), lymph node aspirates (from 182 dogs with swollen lymph nodes), and spleen (from 18 dogs parasitologically proven to have leishmaniasis, after they were euthanized, with the owner's consent), were collected. The number of samples varied from 0 to 300 per prefecture, according to the size of the prefecture and dog population: more than 60 samples (38 prefectures), no samples (11 prefectures).

Serology. Patient and dog sera were tested serologically using anti-human or anti-dog, anti-immunoglobulin $\mathrm{G}$ ( $\mathrm{IgG}$ ) antibodies, respectively, by an indirect immunofluorescent antibody test (IFAT, Leishmania SPOT IF, BioMerieux France). A series of 2-fold serum dilutions, starting from $1 / 40$ were performed and a cut-off titer of $\geq 1 / 160$ was regarded positive for dog sera because most dogs lived in endemic areas. ${ }^{18}$ Any titer $\geq 1 / 160$ was considered suspicious for patients and further diagnostic tests were performed.

PCR. The PCR was carried out on peripheral whole blood, bone marrow and/or skin tissue from patients and on whole blood, lymph node and/or spleen tissue from dogs, according to availability. The QIAamp DNA Blood Mini kit (QIAGEN, Hilden, Germany) and DNeasy Tissue kit (QIAGEN) were used for DNA extraction from blood and tissue, respectively. Primers T2 and B4 were used as described previously with few modifications. ${ }^{17,19}$

Parasite isolation and culture. Whole blood, bone marrow and/or skin tissue were used for the isolation of the parasite from all suspicious patients and blood (with EDTA), lymph node aspirate or spleen tissue from all dogs taking part in the study, according to availability. ${ }^{20}$ The samples were placed over Histopaque 1077 (Sigma, St. Louis, MO) (the tissue samples were first macerated in phosphate buffered saline [PBS]) followed by subsequent washing of the white blood cells (three times in PBS before they were resuspended in RPMI. Half the harvested cells were then placed in NNN and half in RPMI 1640 culture medium, containing $25 \mathrm{mM}$ Hepes buffer, supplemented with $2 \mathrm{mM}$ glutamine (GIBCO Invitrogen, Grand Island, NY), 10\% heatinactivated fetal bovine serum (FBS - GIBCO Invitrogen), $100 \mathrm{IU} / \mathrm{mL}$ penicillin, $100 \mu \mathrm{g} / \mathrm{mL}$ streptomycin (Roche Diag- nostics, Indianapolis, IN), and $5 \%$ filtered human urine, ${ }^{21}$ at $26^{\circ} \mathrm{C}\left( \pm 1^{\circ} \mathrm{C}\right)$. Promastigote cultures were maintained in supplemented RPMI culture medium at $26^{\circ} \mathrm{C}\left( \pm 1^{\circ} \mathrm{C}\right) .{ }^{17,20,21}$

Parasite typing by multilocus enzyme electrophoresis (MLEE, isoenzyme analysis). The parasites, isolated from patients and dogs, were typed by starch gel electrophoresis using 15 enzymatic systems: malate dehydrogenase (MDH), EC 1.1.1.37; malic enzyme, EC 1.1.1.40; isocitrate dehydrogenase, EC 1.1.1.42; 6-phosphogluconate dehydrogenase (PGD), EC 1.1.1.44; glucose-6-phosphate dehydrogenase, EC 1.1.1.49; glutamate dehydrogenase, EC 1.4.1.3; NADH diaphorase (DIA), EC 1.6.2.2; purine nucleoside phosphorylase 1 (NP1), EC 2.4.2.1; NP2, EC 2.4.2*; glutamate-oxaloacetate transaminases 1 and 2 (GOT1 and GOT2), EC 2.6.1.1; phosphoglucomutase, EC 5.4.2.2; fumarate hydratase, EC 4.2.1.2; mannose phosphate isomerase, EC 5.3.1.8; and glucose phosphate isomerase, EC 5.3.1.9. Isoelectrofocusing was used as a technique with greater resolving power for NP1. ${ }^{22}$

Sand fly data. Recent data, recording sandfly presence in Greece, was used from different, already published, studies. ${ }^{15-17}$ In all cases, sampling was carried out during the sand fly season (May to October) using CDC (Centers for Disease Control and Prevention) miniature light traps and A4 paper sticky traps (in some cases mouth and electric powered aspirators were also used). The traps were set for one or more nights at various peridomestic, domestic, or other locations. The insect catch was checked early morning and the trapped sandflies were kept in $70 \%$ ethanol until species identification following morphology-based keys. ${ }^{14,23-26}$ Eighteen locations were studied in $1999-2004,{ }^{15} 8$ locations in $2009-2010,{ }^{16}$ and 9 locations in 2001-2010. ${ }^{17}$

Statistical analysis and mapping of the results. Information, collected from the dog record of each of the 5,772 randomly selected dogs, was used in the statistical analysis to ensure that our sample was representative. For this, 10 dog characteristics were considered and tested by the $\chi^{2}$ test; namely, geographical origin of animal, sampling season, age, symptomatic/ asymptomatic (presence of at least three of any of the classical CanL signs: lymph node swelling, dermatitis furfuracea, alopecia, ulcers, onychogryphosis, epistaxis, ocular lesions, splenomegaly, loss of weight, or unusual tiredness), dog use (hunting, companion, guard dog, etc.), sex, coat shade, length of hair, weight, owned/stray.

Dog seroprevalence was estimated for each prefecture and mapped using the geographical information system software (GIS, Redlands, CA; ArcGIS 10). Associations between dog seropositivity and epidemiological, environmental, and geospatial variables at the place of dog residence were considered using either classical statistics (binary logistic regression model in the SPSS 20; SPSS, Inc., Chicago, IL) or spatial models (spatial binary regression model in the Arcmap 10 [GIS]). These variables were: occurrence of human cases from 2005 to 2010, Leishmania spp. and zymodeme (MON); mean annual wind speed $\left(\mathrm{m} \mathrm{s}^{-1}\right)$, mean annual land surface temperature, mean annual rainfall, and mean annual relative humidity; land cover and altitude $(0-1,000 \mathrm{~m} />1,000 \mathrm{~m})$. Data on wind speed, temperature, rainfall, and relative humidity were obtained from the EDEN DATA WEBSITE of the EU EDEN FP6 project (Emerging Diseases in a changing European environment) and the Hellenic National Meteorological Service. ${ }^{27-29}$ All tests were carried out at a $5 \%$ significance level. 


\section{RESULTS}

Human cases. Between 2005 and 2010, 326 autochthonous human leishmaniasis cases (70.1\% males, $29.9 \%$ females) and one imported CL case were reported in Greece by the HCDCP: $297 \mathrm{VL}$ and $30 \mathrm{CL}$. Of these, the 105 cases were reported to HCDCP by our laboratory and full clinical and epidemiological information (including co-ordinates of place of residence) was available. Information on the place of residence of 255 human VL cases was accessible allowing the mapping of the incidence of the disease (total number of cases per prefecture) (Figure 2).

The 105 cases diagnosed by our laboratory were $0-88$ years of age ( $94 \mathrm{VL}$ and $11 \mathrm{CL}$ ). The VL patients were: $\leq 14$ $(22.9 \%), 15-64(57.5 \%)$, and $\geq 65(19.6 \%)$ years of age. Only one of the VL patients was HIV-positive. All VL patients

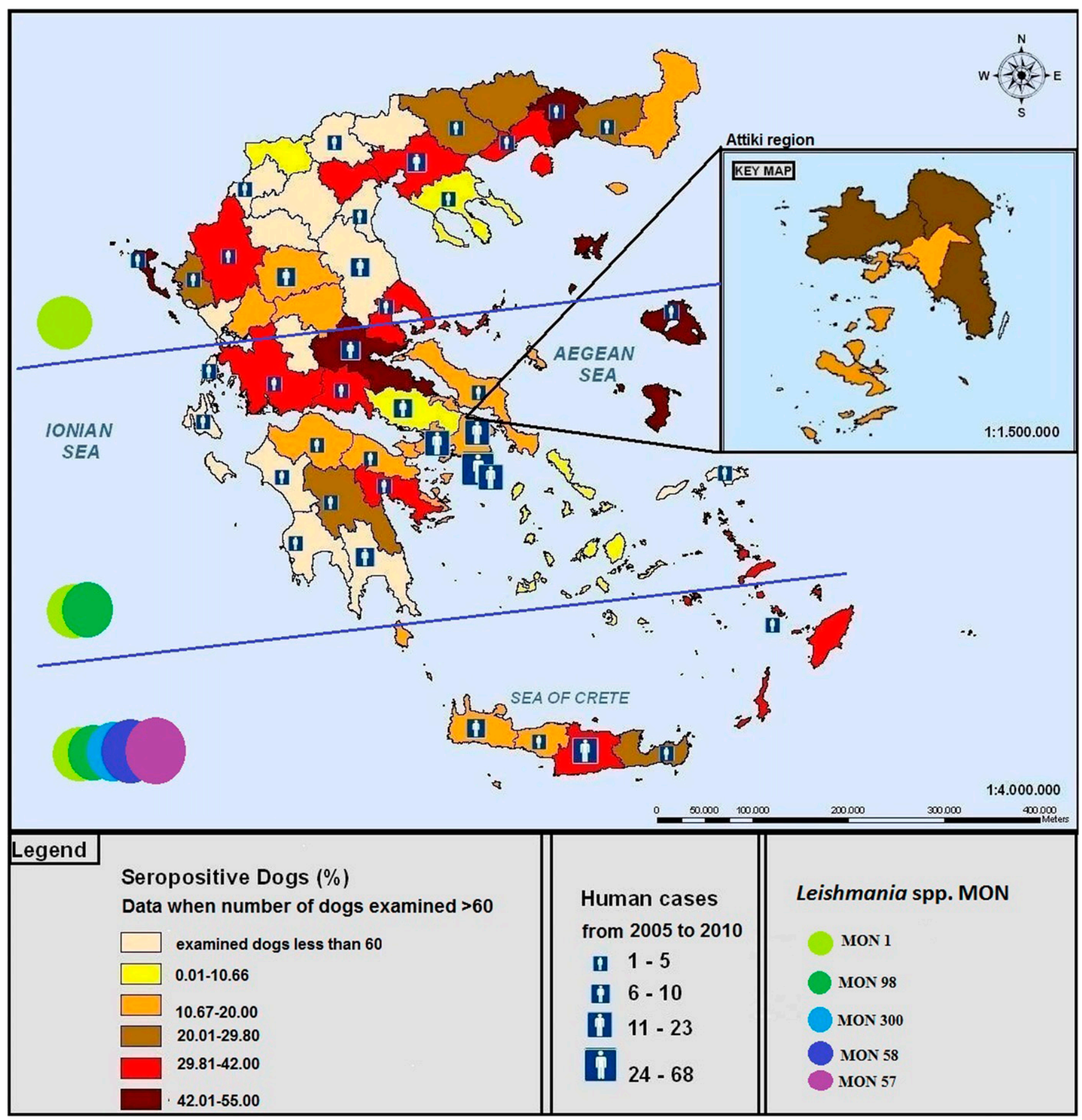

FIGURE 2. Geographical distribution of human leishmaniasis cases in Greece, between 2005 and 2010, in relation to percent dog seropositivity, Leishmania spp., and zymodeme (zymodeme: MON-1, MON-98 L. infantum; MON-300, MON-58, MON-57 L. tropica). (Key map: Attiki region showing the four prefectures). Only data with more than 60 examined dogs per prefecture were taken into account for the mapping of the results. 
were positive by IFAT (titer1/160-1/51,200). The parasite was isolated from 45 patients (from blood [35] and bone marrow sample [44]). Most of the VL patients presented high fever, splenomegaly, and anemia. All VL patients were treated with liposomal amphotericin B and recovered without relapses.

Between 2005 and 2010, 30 cases of CL were reported in Greece. Information was available on 11 patients, reported from our laboratory. All were positive by PCR; only one had antibody response against Leishmania (IFAT titer 1/320); the parasite was isolated from two patients. Seven of the 11 patients were $\geq 60$ years of age. The CL patients had surgical resection of the lesion or cryoablation and only five were treated with Meglumine antimoniate.

Dog epidemiological study. During 2005-2010, 5,772 dogs were examined (2,558 males and 3,214 females; 3,514 owned and 2,258 stray). They were: $\leq 3$ (55\%), 4-6 (25.5\%), 7-9 $(11.6 \%)$, and $\geq 10(7.9 \%)$ years of age; $\leq 10 \mathrm{~kg}(15 \%), 11-$ $20 \mathrm{~kg}(40.5 \%), 21-30 \mathrm{~kg}(31.2 \%)$, and $\geq 30 \mathrm{~kg}(13.3 \%)$. Of the 5,772 dogs, $22.09 \%$ were seropositive (cut-off titer: 1/160): $18.1 \%$ of the females and $24.6 \%$ of the males; $18.7 \%$ of the owned and $27.3 \%$ of the stray dogs. In some regions seropositivity reached $50.2 \%$.

Parasites were isolated from the blood (146), lymph node aspirate (23), bone marrow (8), and spleen (8) of $165 \mathrm{dogs}$, out of the 1,034 tested by culture, 14 of which were healthy looking, seropositive animals. The most common signs in CanL appeared to be lymph node swelling, dermatitis furfuracea, loss of weight, unusual tiredness, alopecia (especially around the eyes), onychogryphosis, and ulceration. Ninety-five seropositive animals had no obvious signs of the disease.

Isolates. Forty seven strains were isolated from human cases: 45 from VL patients, typed as L. infantum (MON-1, 34 isolates [the most common zymodeme in the Mediterranean basin] ${ }^{30,31}$ and MON-98, 11 isolates [a rare zymodeme reported in Egypt $^{32-34}$; Cyprus ${ }^{4}$ and Portugal $]^{35}$ and 2 from CL patients, typed as L. tropica [MON-58, a zymodeme also found in Afghanistan ${ }^{2}$ and MON-300, a zymodeme reported only in Crete, Greece] ${ }^{17}$ ).
The 165 strains isolated from dogs were: $164 \mathrm{~L}$. infantum (136 MON-1 and 28 MON-98) and L. tropica MON-58 (isolated from the blood of a dog with antibody titer1/640).

Statistical analysis and mapping of the results. Our sample, of 5,772 randomly selected animals, was statistically shown to be representative in terms of dog characteristics $(P<0.05$ for all variables with $P<0.001$ for dog sex, dog use, owned/stray). In the statistical analyses and mapping of the results, only data with more than 60 examined dogs per prefecture were considered to have reliable conclusions. The correlation of dog seropositivity in each prefecture with epidemiological, environmental, and spatial factors was estimated so as to test the risk of seropositivity $>20 \%$ in relation to each factor (Table 1). The $>20 \%$ threshold was set because average seropositivity in the country was found to be $22.09 \%$, and $20 \%$ seroprevalence is considered sufficiently high to pose a serious risk of re-emergence of human disease. ${ }^{9,36}$ All environmental factors were found to be highly correlated (positively or negatively) to the seropositivity trends. Areas of low and medium values of mean wind speed $\left(5.9-7.7 \mathrm{~m} \mathrm{~s}^{-1}\right.$ and $7.7-$ $\left.8.6 \mathrm{~m} \mathrm{~s}^{-1}\right)$ presented higher risk $(\mathrm{OR}=4.3[95 \% \mathrm{CI}=1.9-8.7$, $P=0.04] ; \mathrm{OR}=3.2[95 \% \mathrm{CI}=1.04-6.2, P=0.01]$, respectively). On the other hand, as wind speed increased the risk decreased $(\mathrm{OR}=0.8 ; 95 \% \mathrm{CI}=0.6-1.0 ; P=0.002)$. Similarly, areas of high mean land surface temperature presented the highest risk; for instance, areas of $16.01-18.0^{\circ} \mathrm{C}$ had an OR of 79.2 (95\% CI $=76.9-79.3 ; P<0.001)$. Areas of $10.01-13.0^{\circ} \mathrm{C}$ had an OR $=34.1(32.04-35.7 ; P<0.001)$ and areas of 13.01$16.0^{\circ} \mathrm{C}$ presented a higher risk $(\mathrm{OR}=44.09$; $95 \% \mathrm{CI}=39.9$ $46.0 ; P<0.001)$. On the other hand, mean relative humidity presented a proportional correlation. Areas of 74.01-75.6\% mean relative humidity had 27.75 times higher probability of $>20 \%$ seropositivity $(95 \% \mathrm{CI}=25.0-29.0, P<0.001)$ compared with the areas of $72.01-74.0 \%$ and $67.01-72.0 \%$ relative humidity $(\mathrm{OR}=1.4 ; 95 \% \mathrm{CI}=1.2-1.2 ; P=0.01$ and $\mathrm{OR}=1.1$; $95 \% \mathrm{CI}=1.01-1.2 ; P=0.003$, respectively). Finally, regions that had a mean annual rainfall of $60.01-100.0 \mathrm{~mm}$ presented

TABLE 1

Risk of dog seropositivity to be $>20 \%$ in relation to environmental risk factors*

\begin{tabular}{|c|c|c|c|}
\hline \multirow[b]{2}{*}{ Environmental factor } & \multirow{2}{*}{$\frac{\text { Number of prefectures with }>20 \% \text { seropositivity }}{\mathrm{N}(\%)}$} & \multirow[b]{2}{*}{ Odds ratio $(95 \% \mathrm{CI})$} & \multirow[b]{2}{*}{$P$ value } \\
\hline & & & \\
\hline Mean wind speed $\left(\mathrm{m} \mathrm{s}^{-1}\right)$ Prefectures examined 49 & & & $<0.001$ \\
\hline$<5.9$ & $14(28.5)$ & 1 & - \\
\hline $5.9-7.7$ & $18(36.7)$ & $4.3(1.9-8.7)$ & 0.04 \\
\hline $7.7-8.6$ & $15(30.6)$ & $3.2(1.04-6.2)$ & 0.01 \\
\hline$>8.6$ & $2(4.2)$ & $0.8(0.6-1.0)$ & 0.002 \\
\hline Mean - land surface temperature $\left({ }^{\circ} \mathrm{C}\right)$ Prefectures examined 49 & & & $<0.001$ \\
\hline $8.6-10.0$ & $6(12.2)$ & 1 & - \\
\hline $10.01-13.0$ & $13(26.5)$ & $34.1(32.0-35.7)$ & $<0.001$ \\
\hline $13.01-16.0$ & $15(30.6)$ & $44.09(39.9-46.0)$ & $<0.001$ \\
\hline $16.01-18.0$ & $13(26.5)$ & $79.2(76.9-79.3)$ & $<0.001$ \\
\hline $18.0-19.7$ & $2(4.1)$ & $50.1(49.2-52.9)$ & 0.016 \\
\hline \multicolumn{4}{|l|}{ Mean annual rainfall $(\mathrm{mm})$ Prefectures examined 24} \\
\hline $10-30.0$ & $14(59.2)$ & 1 & - \\
\hline $30.01-60.0$ & $4(20.4)$ & $1.2(1.1-1.8)$ & $<0.001$ \\
\hline $60.01-100.0$ & $2(10.2)$ & $2.3(1.8-3.1)$ & 0.045 \\
\hline $100.01-138.0$ & $4(20.4)$ & $0.8(0.5-1.0)$ & $<0.001$ \\
\hline \multicolumn{4}{|l|}{ Mean - relative humidity (\%) Prefectures examined 49} \\
\hline $65.8-67.0$ & $6(12.2)$ & 1 & - \\
\hline $67.01-72.0$ & $15(30.6)$ & $1.1(1.01-1.2)$ & 0.0034 \\
\hline $72.01-74.0$ & $15(30.6)$ & $1.4(1.2-1.2)$ & 0.010 \\
\hline $74.01-75.6$ & $13(26.5)$ & $27.8(25.004-29.0)$ & $<0.001$ \\
\hline
\end{tabular}

* Only data with more than 60 examined dogs per prefecture were taken into account for the analysis of the results. 
TABLE 2

Risk of dog seropositivity to be $>20 \%$, in relation to geospatial and epidemiological risk factors*

\begin{tabular}{lcc}
\hline Geospatial and epidemiological factors & Odds ratio $(95 \% \mathrm{CI})$ & $P_{\text {trend }}$ \\
\hline Land cover & & \\
Water bodies & $1.4(1.01-1.6)$ & $<0.001$ \\
Agricultural areas & $2.2(2.0-2.9)$ & \\
Semi-natural areas & $1.2(1.01-1.1)$ & \\
Open spaces with no vegetation & $0.8(0.6-1.0)$ & \\
Artificial surfaces & $0.02(0.005-0.1)$ & \\
Altitude (m) & 1 & $<0.001$ \\
$>1000$ & $3.0(1.3-4.4)$ & \\
$0-1000$ & & \\
\hline * Only data with more than 60 examined dogs per prefecture were taken into account for \\
the analysis of the results.
\end{tabular}

the highest risk $(\mathrm{OR}=2.3,95 \% \mathrm{CI}=1.8-3.06, P=-0.04)$. Spatial factors (land cover and altitude) were found to be of very high statistical significance $(P<0.001$ for both variables) (Table 2). As far as land cover is concerned, presence of water bodies in an area $(\mathrm{OR}=1.4,95 \% \mathrm{CI}=1.01-1.6)$, agricultural areas $(\mathrm{OR}=2.2,95 \% \mathrm{CI}=2.0-2.9)$ and semi-natural areas $(\mathrm{OR}=1.2,95 \% \mathrm{CI}=1.01-1.1)$ presented the highest risk. On the other hand, open spaces with no vegetation $(\mathrm{OR}=0.8$, $95 \% \mathrm{CI}=0.6-1.0)$ and artificial surfaces $(\mathrm{OR}=0.02,95 \%$ $\mathrm{CI}=0.005-0.1)$ did not seem to favor dog seropositivity. Furthermore, areas of $0-1,000 \mathrm{~m}$ altitude were estimated to present higher risk than those of $>1,000 \mathrm{~m}(\mathrm{OR}=3.0 ; 95 \%$ $\mathrm{CI}=1.3-4.4 ; P<0.001)$.

\section{DISCUSSION}

Leishmaniasis constitutes a public health problem in Europe, where it is endemic in many parts and recently reported in new geographical regions. ${ }^{1,37,38}$ During the last 35 years, the disease has spread to most parts of Greece because of $L$. infantum, causing VL and CanL and to L. tropica, causing CL ${ }^{39-41}$ During 2005-2010, 327 human leishmaniasis cases (297 VL and 30 CL) were reported in the country. Autochthonous human VL cases were reported in 41/54 prefectures (Figure 2) the majority in the Attiki prefecture (mostly in the city of Athens, which is an endemic VL area) ${ }^{42}$ with the maximum number $(72)$ in the year 2007 (HCDCP). The majority of the cases were adults, between 15 and 88 years of age ( $77.1 \%$ of the cases); males $(70.1 \%)$, and only one was HIV positive. All VL patients tested were seropositive, yet asymptomatic people from endemic areas may present transient parasitemia and antileishmanial antibody response ${ }^{43-45}$ making diagnosis of active VL difficult. ${ }^{46}$ Such healthy immunocompetent people may later develop VL if they suffer immunosuppression, mainly caused by HIV. Such a co-infection will aid in changing the epidemiology of the disease because these patients are infective to sandflies. ${ }^{47}$ At the same time, other forms of immunosuppression, like organ transplantation and also mild immunosuppression, like psychological stress and malnutrition, may allow this opportunistic parasite to cause disease. Five cases of VL after psychological stress and one after fasting for religious purposes were presented in our hospital. ${ }^{17}$ The drug used in treating the majority of VL cases in Greece is liposomal amphotericin B, which appears to be efficient and no relapses have been reported.

Leishmania infantum MON-1, found predominantly in the Mediterranean Basin ( $90 \%$ of the typed isolates), ${ }^{31,48}$ was

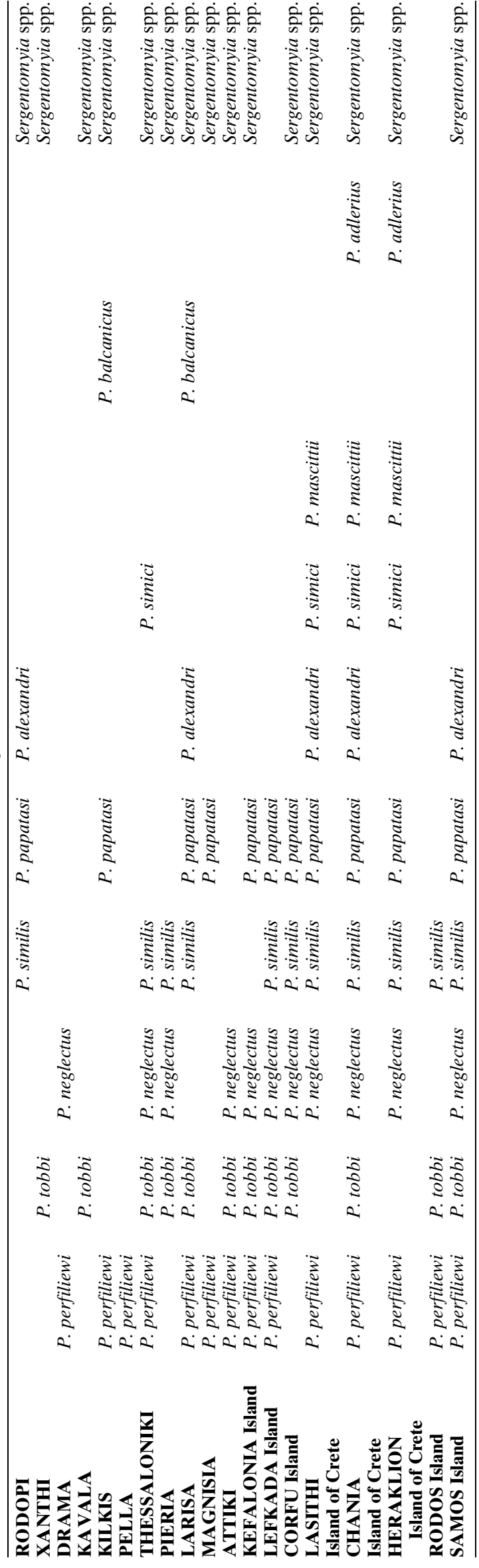


present in all parts of the country in patients and dogs $(75.6 \%$ of the VL isolates; $82.9 \%$ of the CanL isolates) (Figure 2), the rare zymodeme MON-98 was found in patients $(24.4 \%)$ and dogs $(17.1 \%)$ but only in the central and southern parts of the country (Figure 2). The dermatotropic L. tropica is shown only in Crete (Figure 2), despite the fact that it is also reported from the Ionian Islands, because no detailed records and biological material for parasite isolation were available from CL patients from other hospitals in Greece during the study period.

The sample, of 5,772 dogs, was shown to be statistically representative in terms of geographical spread and dog characteristics. Average seroprevalence was $22.09 \%$ (ranging from $6.5 \%$ in western Macedonia, to $50.2 \%$ in the island of Corfu) (Figure 2) and seropositive dogs were found in nearly all prefectures from which dog samples were available (41/43) indicating the spread of the disease in the entire country. The presence of seropositive dogs in the 11 prefectures from which no samples were available cannot be excluded because, neighboring prefectures with similar bioclimatic and ecological characteristics (that favor sandfly survival and development), presented dog seropositivity higher than $10 \%$ (Figure 2 ). This is supported by the statistical analysis of the results showing a significant association of high CanL seroprevalence (> 20\%) with environmental, spatial, and epidemiological factors that suit the ecological requirements of these nocturnal sandfly vectors (Table 1$){ }^{49}$

It is known that a great proportion of dogs, residing in endemic areas, are infected even if they remain asymptomatic, or seronegative. ${ }^{50}$ Serology detects most of the symptomatic and a proportion of the asymptomatic dogs that may be as high as $50 \%$ of the seropositive canine population. ${ }^{51-53}$ In our study, 95 of the seropositive dogs were without any obvious leishmaniasis signs and the parasite was isolated from 14 such animals. Seroprevalence in dogs in an area is believed to be between the prevalence of the disease and that of the infection. ${ }^{11}$ The important issue, however, is the ability of positive, but asymptomatic, dogs to transmit the parasite to the sandfly vector. ${ }^{54}$ Yet, because the majority of the PCR positive dogs were dogs with at least three CanL signs $(P<0.0001)$, symptomatic dogs are more likely to infect sandflies. ${ }^{17}$ Dogs sleeping outside (stray, guard, hunting dogs) are at higher risk of getting infected (seropositive; $P<0.001$ ) and therefore to infect sandflies.

Controlling the disease in the dog population is the best way to reduce the risk of infection in the human population; therefore, detailed and accurate studies are necessary for the implementation of targeted control measures. Often, however, there are limitations in such studies, especially regarding standardized procedures in conducting canine surveys and serological testing.

$P$. perfiliewi (found in 14 of 19 prefectures where sandfly trapping has been carried out), P. tobbi (in 12 of 19), and P. neglectus (in 11 of 19) (Table 3, Figure 3), all known vectors of L. infantum in the Mediterranean basin, were reported in all longitudes of the country. Interestingly, $P$. perfiliewi was the only sandfly species found in Pella prefecture where one autochthonous human VL case was reported (Figures 1 and 3). $P$. similis was found in 11 of 19 prefectures studied. This species has often been misidentified as $P$. sergenti, sister species of $P$. similis, implying a similar vector capability in relation to Leishmania species. ${ }^{55}$ This species is believed to be the potential vector of $L$. tropica in Crete, ${ }^{17}$ a fact that needs further investigation. $P$. similis was found in the Ionian Islands and Crete where $\mathrm{CL}$ is widespread but also in areas on the coast of the Aegean Sea (Figures 1 and 3) where CL cases have never been reported. These areas should be considered high

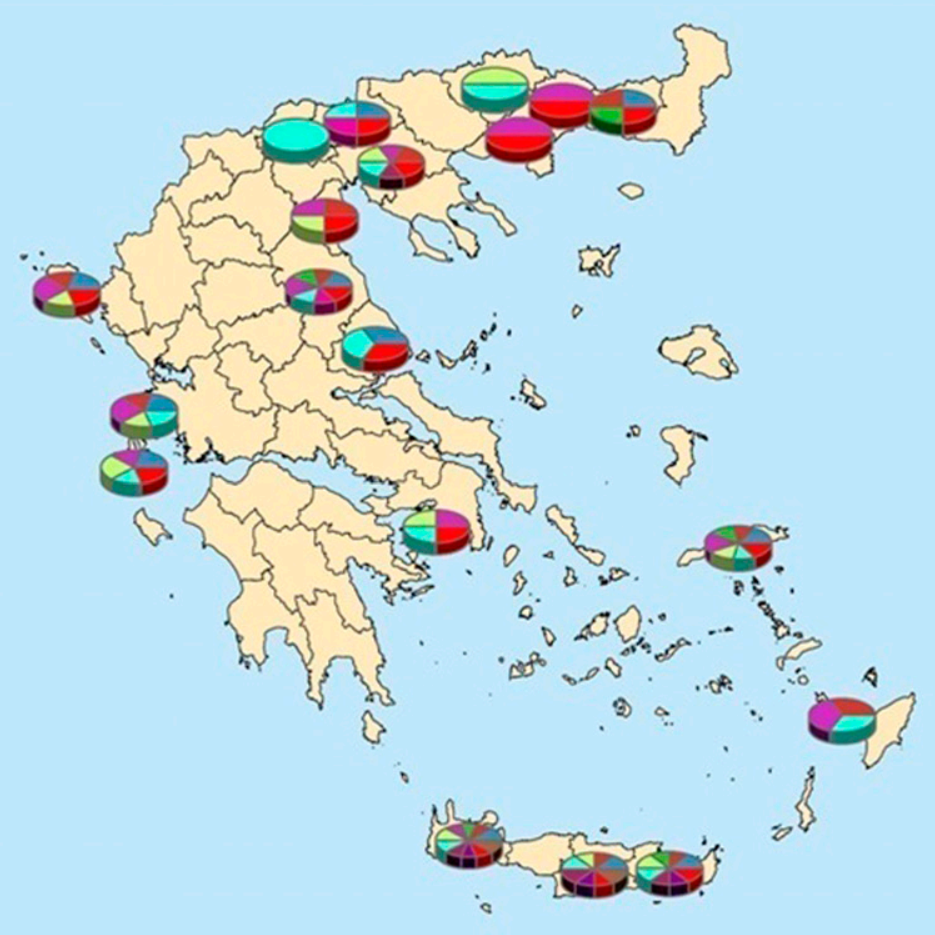

Legend

SANDFLIES

\begin{tabular}{|l} 
P. tobbi \\
P. perfiliewi \\
\hline P. neglectus \\
P. papatasi \\
P. mascitti \\
P. similis \\
P. alexandri \\
P. alderius \\
P. balcanicus \\
P. simici \\
Sergentomyia spp.
\end{tabular}

FIGURE 3. Recorded presence of phlebotomine sandflies in Greece. ${ }^{15-17}$ 
risk for the introduction of this anthroponotic disease because the vector is present and infected people may start the transmission cycle of the parasite. P. papatasi was found in 11 of 19 prefectures studied, in northern and central Greece as well as the Ionian Islands, Crete, and Samos. P. papatasi is able to transmit $L$. major causing zoonotic cutaneous leishmaniasis using certain rodents as reservoir hosts. This form of leishmaniasis is found in northern Africa and the Middle East. More sandfly studies are needed, covering all prefectures, before a reliable conclusion can be reached on the risk of transmission of L. infantum and L. tropica in all prefectures. Despite the fact that $80 \%$ of the terrain of Greece is mountainous, mainly the central part dividing the country into two (Figure 1), the same Phlebotomus spp. were found in the eastern and the western parts (Figure 3, Table 3).

The CL, caused by L. tropica, appears to be re-emerging, after being latent for 35 years, in some areas. ${ }^{39-41}$ Strains, isolated from CL patients from the Ionian Islands and Crete in the early 1980 s, were typed as L. tropica MON-57. ${ }^{56}$ Yet, zymodeme MON-300, found for the first time in Crete, was isolated from a 64-year-old woman who reported that she was infected at childhood, like a great number of her fellow villagers. ${ }^{17}$ The woman relapsed three times and although she was treated with Meglumine antimoniate, she was never cleared from the parasite. Seven of the CL patients from Crete were $\geq 60$ years of age and all reported having lesions, known as the "Chaniotico spyri" ("the skin lesion found in the area of Chania," Crete), at childhood. Possibly changes in the immune system of these people, caused by age, has allowed the parasite to become activated and cause new lesions. L. tropica MON-58 was also isolated in Crete from a young Afghan refugee and a dog in the area where the boy worked in the summer (Ntais and others, unpublished data). It is evident that a polymorphism of L. tropica exists in Crete and that new species and strains may be introduced in this closed ecosystem (like L. donovani in Cyprus) ${ }^{5}$ where 10 Phlebotomus spp. are well established (Figure 3, Table 3) and may be able to support their introduction and spread. We believe that more CL patients are found in Greece because people, being aware of its benign nature, do not consider it necessary to visit the doctor, especially the elderly. Furthermore, if they visit private dermatologists the disease may not be reported and so measures for monitoring the disease are not taken. As a result, such patients, who can infect sandflies, become the source of this anthroponotic pathogen. Although sandfly vectors implicated in the transmission of each parasite are different, there are 13 known sandfly species in Greece, 10 of which belong to the medically important genus of Phlebotomus and 3 to Sergentomyia, ${ }^{13-17}$ and are widely spread in the country (Figure 3, Table 3). The close association of the vectors with human habitation, especially in rural areas, ensures transmission of $L$. infantum from infected dogs to humans. Most of the dogs studied lived outdoors (78.7\%), a factor believed to favor the rate of infection. ${ }^{57}$ The danger of exchange of genetic material between these species and zymodeme variants found in a closed ecosystem, resulting in new hybrids, is a possibility. ${ }^{58,59}$

It is evident that during the last 35 years, leishmaniasis cases in humans and dogs are increasing in numbers and are spreading geographically, favored by the changing conditions: like the movement of people and animals, the buildup of vector populations caused by changing climatic and environ- mental conditions, and changes in animal husbandry practices. Studies are undertaken to foresee changing conditions and how they affect parasite spread (EU EDEN fp6 and EDENext fp7 programs) ${ }^{60}$ so that targeted measures can be taken to prevent the parasite's arrival, settlement, and spread in new areas. $^{28}$

Received February 6, 2013. Accepted for publication May 28, 2013. Published online September 23, 2013.

Acknowledgments: We thank Jean-Pierre Dedet for advice and for revising the manuscript, the 63 veterinarians who took part in the project; provided dog samples and completed the dog records, and PESYDAP for its valuable collaboration. This work was partially funded by the University of Crete, Special Account for Research.

Financial support: This study was partially funded by EU grant FP7-261504 EDENext and is catalogued by the EDENext Steering Committee as EDENext120 (http://www.edenext.eu).

Disclaimer: The contents of this publication are the sole responsibility of the authors and do not necessarily reflect the views of the European Commission.

Authors' addresses: Pantelis Ntais, Dimitra Sifaki-Pistola, Ippokratis Messaritakis, and Maria Antoniou, Laboratory of Clinical Bacteriology, Parasitology, Zoonoses and Geographical Medicine, Faculty of Medicine, University of Crete, Crete, Greece, E-mails: pntais@yahoo .com, spdimi11@gmail.com, imessar@edu.med.uoc.gr, and antoniou@ med.uoc.gr. Vasiliki Christodoulou, Laboratory of Clinical Bacteriology, Parasitology, Zoonoses and Geographical Medicine, Faculty of Medicine, University of Crete, Crete, Greece and Veterinary Services of Cyprus, Nicosia, Cyprus, E-mail: vchristod@edu.med.uoc.gr. Francine Pratlong, Université Montpellier 1, Laboratoire de Parasitologie, Centre National de Référence des Leishmania, CHU de Montpellier and UMR 2724 GEMI (IRD-CNRS-UM1), Montpellier, France, E-mail: f-pratlong@chu-montpellier.fr. George Poupalos, Mesologgiou 64, Kamatero, Athens, E-mail: gpoupalos@yahoo.com.

\section{REFERENCES}

1. Alvar J, Velez ID, Bern C, Herrero M, Desjeux P, Cano J, Jannin J, den Boer M, 2012. Leishmaniasis worldwide and global estimates of its incidence. PLOS ONE 7: e35671.

2. Pratlong F, Dereure J, Ravel C, Lami P, Balard Y, Serres G, Lanotte G, Rioux JA, Dedet JP, 2009. Geographical distribution and epidemiological features of Old World cutaneous leishmaniasis foci, based on the isoenzyme analysis of 1048 strains. Trop Med Int Health 14: 1071-1085.

3. World Health Organization, 2009. Leishmaniasis: background information. A brief history of the disease. Available at: http:// www.who.int/leishmaniasis/en. Accessed December 11, 2011.

4. Mazeris A, Soteriadou K, Dedet JP, Haralambous C, Tsatsaris A, Moschandreas J, Messaritakis I, Christodoulou V, Papadopoulos B, Ivovic V, Pratlong F, Loucaides F, Antoniou M, 2010. Leishmaniases and the Cyprus paradox. Am J Trop Med Hyg 82: 441-448.

5. Antoniou M, Haralambous C, Mazeris A, Pratlong F, Dedet JP, Soteriadou K, 2008. Leishmania donovani leishmaniasis in Cyprus. Lancet Infect Dis 8: 6-7.

6. Maroli M, Rossi L, Baldelli R, Capelli G, Ferroglio E, Genchi C, Gramiccia M, Mortarino M, Pietrobelli M, Gradoni L, 2008. The northward spread of leishmaniasis in Italy: evidence from retrospective and ongoing studies on the canine reservoir and phlebotomine vectors. Trop Med Int Health 13: 256-264.

7. Bogdan C, Schonian G, Bañuls AL, Hide M, Pratlong F, Lorenz E, Röllinghoff M, Mertens R, 2001. Visceral leishmaniasis in a German child who had never entered a known endemic area: case report and review of the literature. Clin Infect Dis 32: 302-306.

8. Tánczos B, Balogh N, Király L, Biksi I, Szeredi L, Gyurkovsky M, Scalone A, Fiorentino E, Gramiccia M, Farkas R, 2012. First record of autochthonous canine leishmaniasis in Hungary. Vector Borne Zoonotic Dis 12: 588-594. 
9. Ready PD, 2008. Leishmaniasis emergence and climate change. Rev Sci Tech 27: 399-412.

10. Dujardin JC, 2006. Risk factors in the spread of leishmaniases: towards integrated monitoring? Trends Parasitol 22: 4-6.

11. Gradoni L, 1999. Epizootiology of canine leishmaniasis in southern Europe. Killick-Kendrick R, eds. Canine leishmaniasis: an update. Proceedings of the Canine Leishmaniasis forum, Barcelona, Spain. Wiesbaden, Germany: Hoechst Roussel Vet, 32-39.

12. Caminopetros J, 1934. New epidemiological and experimental data on leishmaniasis in Greece. Medical Athens 43: 653-659.

13. Léger N, Gramiccia M, Gradoni L, Madulo-Leblond G, Pesson B, Ferté H, Boulanger N, Killick-Kendrick R, Killick-Kendrick M, 1988. Isolation and typing of Leishmania infantum from Phlebotomus neglectus on the island of Corfu, Greece. Trans $R$ Soc Trop Med Hyg 82: 419-420.

14. Léger N, Pesson B, Madulo-Leblond G, Ferté H, Tselentis Y, Antoniou M, 1993. Les phlebotomes de Crete. Resultats d'une enquete entomologique effectuee en Juillet 1988 et Aout 1989. Biologia Gallo-hellenica 20: 135-143.

15. Ivovic V, Patakakis M, Tselentis Y, Chaniotis B, 2007. Faunistic study of sandflies in Greece. Med Vet Entomol 21: 121-124.

16. Xanthopoulou K, Anagnostou V, Ivovic V, Djurkovic-Djakovic O, Rogozi E, Sotiraki S, Papa A, 2011. Distribution of sandflies (Diptera, Psychodidae) in two Ionian Islands and northern Greece. Vector Borne Zoonotic Dis 11: 1591-1594.

17. Christodoulou V, Antoniou M, Ntais P, Messaritakis I, Ivovic V, Dedet JP, Pratlong F, Dvorak V, Tselentis Y, 2012. Re-emergence of visceral and cutaneous leishmaniasis in the Greek Island of Crete. Vector Borne Zoonotic Dis 12: 214-222.

18. Ferroglio E, Trisciuoglio A, Gastaldo S, Mignone W, Delle Piane M, 2002. Comparison of ELISA, IFAT, and Western blot for the serological diagnosis of Leishmania infantum infection in dog. Parassitologia 44 (Suppl1): 64.

19. Minodier P, Piarroux R, Gambarelli F, Joblet C, Dumon H, 1997. Rapid identification of causative species in patients with Old World leishmaniasis. J Clin Microbiol 32: 746-749.

20. World Health Organization, 1991. Basic Laboratory Methods in Medical Parasitology. Geneva: WHO.

21. Howard MK, Pharoah MM, Ashall F, Miles MA, 1991. Human urine stimulates growth of Leishmania in vitro. Trans $R$ Soc Trop Med Hyg 85: 477-479.

22. Piarroux R, Trouvé V, Pratlong F, Martini A, Lambert M, Rioux JA, 1994. The use of isoelectric focusing on polyacrylamide gel for the enzymatic analysis of Old World Leishmania species. Trans $R$ Soc Trop Med Hyg 88: 475-478.

23. Theodor O, 1958. Psychodidae-Phlebotominae. Linder E, ed. Die Fliegen der Palaearktischen Region. Stuttgart: E. Schweitzer bartsche Verlagsbuchhandlung, $9 c: 1-55$.

24. Perfiliev P, 1966. Fauna of USSR. Diptera: Phlebotomidae (sand flies). Akademia Nauk SSSR, Jerusalem: Israel Programme for Scientific Translations, $1-383$

25. Lewis D, 1982. A taxonomic review of the genus Phlebotomus (Diptera: Psychodidae). Bull Br Mus Nat Hist 45: 171-209.

26. Depaquit J, Léger N, Ferté H, Rioux JA, Gantier JC, Michaelides A, Economides P, 2001. Les phlebotomes de l'ile de Chypre. III-Inventaire faunistique. Parasite Immunol 8: 11-20.

27. EDENext Data Portal, 2010. Available at: http://www.edendatasite .com/.

28. About EDEN, (Emerging Diseases in a changing European eNvironment). Available at: http:www.eden-fp6project.net.

29. Hellenic National Metereological Service. Available at: http://www .hnms.gr/hnms/greek/index_html. Accessed December 15, 2010.

30. Pratlong F, Lami P, Ravel C, Balard Y, Dereure J, Serres G, El Baidouri F, Dedet JP, 2012. Geographical distribution and epidemiological features of Old World Leishmania infantum and $L$. donovani foci, based on the isoenzyme analysis of 2.277 strains. Parasitol 12: 1-12.

31. Ait-Oudhia K, Harrat Z, Benikhlef R, Dedet JP, Pratlong F, 2011. Isoenzymatic polymorphism of Leishmania infantum causing canine leishmaniasis: a review including 996 strains of the Mediterranean area, with special reference to Algeria. Acta Trop 118: 80-86.

32. Doha S, Shehata MG, 1992. Leishmania infantum MON-98 isolated from naturally infected Phlebotomus langeroni (Diptera: Psychodidae) in El Agamy, Egypt. J Med Entomol 29: 891-893.
33. Shetata M, el Sawaf B, el Said S, Doha S, el Hosary S, Kamal H, Dereure J, Pratlong F, Rioux JA, 1990. Leishmania infantum MON-98 isolated from dogs in El Agamy, Egypt. Trans $R$ Soc Trop Med Hyg 84: 227-228.

34. Youssef M, Shehata MG, el Sawaf BM, Boulos L, Pratlong F, Amer M, Rioux JA, 1989. Leishmania infantum MON-98, a new zymodem isolated from human visceral leishmaniasis in Egypt (El Agamy). Ann Parasitol Hum Comp 64: 152-153.

35. Cardoso L, Santos H, Cordeiro-da-Silva A, Pratlong F, Dedet JP, Rodrigues M, 2002. Leishmania infantum MON-98: infection in a dog from Alto Douro, Portugal. Acta Trop 83: 83-85.

36. Dujardin JC, Campino L, Canavate C, Dedet JP, Gradoni L, Soteriadou K, Mazeris A, Ozbel Y, Boelaert M, 2008. Spread of vector-borne diseases and neglect of leishmaniasis, Europe. Emerg Infect Dis 14: 1013-1018.

37. Desjeux P, 1996. Leishmaniasis: public health aspects and control. Clin Dermatol 14: 417-423.

38. World Health Organization, 2004. The vector-borne human infections of Europe, their distribution and burden on Public Health, "Sandfly-borne diseases-leishmaniasis," 27-29.

39. Garifallou A, Schnur LF, Stratigos JD, Hadziandoniou M, Savigos M, Stavrianeas N, Sérié C, 1984. Leishmaniasis in Greece II. Isolation and identification of the parasite causing cutaneous leishmaniasis in man. Ann Trop Med Parasitol 78: 369-375.

40. Tzamouranis N, Schnur LF, Garifallou A, Pateraki E, Sérié C, 1984. Leishmaniasis in Greece I. Isolation and identification of the parasite causing human and canine visceral leishmaniasis. Ann Trop Med Parasitol 78: 363-368.

41. Frank C, Hadziandoniou M, Pratlong F, Garifallou A, Rioux JA, 1993. Leishmania tropica and Leishmania infantum responsible for cutaneous leishmaniasis in Greece: sixteen autochthonous cases. Trans $R$ Soc Trop 87: 184-185.

42. Tselentis Y, Gikas A, Chaniotis B, 1994. Kala-azar in Athens basin. Lancet 343: 1635.

43. Papadopoulou C, Kostoula A, Dimitriou D, Panagiou A, Bobojianni C, Antoniades G, 2005. Human and canine leishmaniasis in asymptomatic and symptomatic population in northwestern Greece. J Infect 50: 53-60.

44. Badaro R, Reed SG, Barral A, Orge G, Jones TC, 1986. Evaluation of the micro enzyme-linked immunosorbent assay (ELISA) for antibodies in American visceral leishmaniasis: antigen selection for detection of infection-specific responses. Am J Trop Med Hyg 35: 72-78.

45. Chandra J, Ravi RN, Mittal SK, Sharma D, 1991. Kala-Azar without hepatosplenomegaly. Indian Pediatr 28: 1185-1186.

46. Luz K, da Silva VO, Gomes EM, Machado FC, Araujo MA, Fonseca HE, Freire TC, d'Almeida JB, Palatnik M, Palatnik-de Sousa CB, 1997. Prevalence of anti-Leishmania donovani antibody among Brazilian blood donors and multiply transfused hemodialysis patients. Am J Trop Med Hyg 57: $168-171$.

47. Desjeux P, Alvar J, 2003. Leishmania/HIV co-infections: epidemiology in Europe. Ann Trop Med Parasitol 97: 3-15.

48. Pratlong F, Lami P, Ravel C, Balard Y, Dereure J, Serres G, El Baidouri F, Dedet JP, 2013. Geographical distribution and epidemiological features of Old World Leishmania infantum and Leishmania donovani foci, based on the isoenzyme analysis of 2277 strains. Parasitology 140: 423-434.

49. Franco AO, Davies CR, Mylne A, Dedet JP, Gallego M, Ballart C, Gramiccia M, Gradoni L, Molina R, Galvez R, MorillasMarquez F, Baron-Lopez S, Pires CA, Afonso MO, Ready PD, Cox J, 2011. Predicting the distribution of canine leishmaniasis in western Europe based on environmental variables. Parasitology 14: 1-14.

50. Ciaramella P, Oliva G, Luna RD, Gradoni L, Ambrosio R, Cortese L, Scalone A, Persechino A, 1997. A retrospective clinical study of canine leishmaniasis in 150 dogs naturally infected by Leishmania infantum. Vet Rec 141: 539-543.

51. Leontides L, Saridomichelakis MN, Billinis C, Kontos V, Koutinas AF, Galatos AD, Mylonakis ME, 2002. A cross-sectional study of Leishmania spp. infection in clinically healthy dogs with polymerase chain reaction and serology in Greece. Vet Parasitol 109: 19-27.

52. Solano-Gallego L, Morell P, Arboix M, Alberola J, Ferrer L, 2001. Prevalence of Leishmania infantum infection in dogs 
living in an area of canine leishmaniasis endemicity using PCR on several tissues and serology. J Clin Microbiol 39: 560-563.

53. Fisa R, Gallego M, Castillejo S, Aisa MJ, Serra T, Riera C, Carrio J, Gallego J, Portus M, 1999. Epidemiology of canine leishmaniasis in Catalonia (Spain) the example of the Priorat focus. Vet Parasitol 83: 87-97.

54. Killick-Kendrick R, 1990. Phlebotomine vectors of the leishmaniases: a review. Med Vet Entomol 4: 1-24.

55. Depaquit J, Ferté H, Léger N, Lefranc F, Alves-Pires C, Hanafi H, Maroli M, Morillas-Marquez F, Rioux JA, Svobodova M, Volf $\mathrm{P}, 2002$. ITS 2 sequences heterogeneity in Phlebotomus sergenti and Phlebotomus similis (Diptera, Psychodidae): possible consequences in their ability to transmit Leishmania tropica. Int $J$ Parasitol 32: 1123-1131.

56. Rioux J, Lanotte G, Serres E, Pratlong F, Bastien P, Perieres J, 1990. Taxonomy of Leishmania. Use of isoenzymes. Suggestions for a new classification. Ann Parasitol Hum Comp 65: $111-125$.

57. Zaffaroni E, Rubaudo L, Lanfranchi P, Mignone W, 1999. Epidemiological patterns of canine leishmaniasis [correction of leishmaniosis] in western Liguria (Italy). Vet Parasitol 81: 11-19.

58. Ravel C, Cortes S, Pratlong F, Morio F, Dedet JP, Campino L, 2006. First report of genetic hybrids between two very divergent Leishmania species: Leishmania infantum and Leishmania major. Int J Parasitol 36: 1383-1388.

59. Volf P, Benkova I, Myskova J, Sadlova J, Campino L, Ravel C, 2007. Increased transmission potential of Leishmania majorl Leishmania infantum hybrids. Int J Parasitol 37: 589-593.

60. Cirad, 2011. EDENext. Biology and Control of Vector-Borne Infections in Europe. Available at: http://www.edenext.eu. Accessed August 10, 2012. 